\title{
Vergleichende Untersuchungen reduktometrischer und enzymatischer Harnsäurebestimmungen
}

\author{
Von Chr. Bünemann und J. D. Kruse-Jarres \\ Klinisch-Chemisches Labor der Chirurg. Univ. Klinik Freiburg i. Br.
}

(Eingegangen am 19. März 1973/30. Mai 1973)

\begin{abstract}
Es werden 6 Methoden zur Harnsäurebestimmung miteinander verglichen: die auf der Reduktion von Phosphorwolframsäure basierenden Tests AccUric Macro bzw. Micro und dic Phosphorwolframat-Methode nach Uranylacetat-Enteiweißung, sowic dic enzymatische Bestimmung durch dic direkte Messung des $\mathrm{O}_{2}$-Vcrbrauchs, der UV-Test mit Uricase und die colorimetrische Uricase-Katalase-Methode. Hinsichtlich der Qualität der crzielten Ergebnisse bestchen zum Teil großc Unterschicdc. Der bisher als Standard-Referenz-Methode dienende sehr zuverlässige Uricase-UV-Test wird von der Uricase-Katalase-Methode hinsichtlich der besseren Reproduzierbarkeit und größeren Stabilität noch übertroffen.
\end{abstract}

\section{Comparative studies on reductometric and enzymic methods for the determination of uric acid}

Six methods for the determination of uric acid were compared: the macro and micro AccUric tests based on the reduction of phosphotungstic acid; the phosphotungstic method after deproteinization with uranyl acetate, the enzymic determination by direct measurement of $\mathrm{O}_{2}$ consumption; the UV-test with uricase; and the colorimetric uricasc-catalase method. There were some large differences in the quality of the results. With respect to reproducibility and stability, the very reliable uricase UV-test, which has hitherto been used as a standard reference, was surpassed by the uricase-catalase method.

Klinische Notwendigkeit bei der anscheinend stetig wachsenden $\mathrm{Zahl}$ von Gichtkranken sowie unterschiedliche Bewertung von Ergebnissen verschiedener Bestimmungen der Harnsäure im Serum haben uns bewogen, die zur Zeit gebräuchlichsten Methoden miteinander $z u$ vergleichen. Anla $B$ waren stark divergierende Darstellungen von Untersuchern und Herstellern über die Güte ihrer Methode. Die verschiedenen Auffassungen dokumentieren sich im Ringversuch der Deutschen Gesellschaft für Klinische Chemie vom 19. 2. 1973, in welchem u. a. auch der Parameter Harnsäure von 99 Laboratorien untersucht wurde. Abgesehen von Analysen enzymatischer Bestandteile steht die Harnsäurebestimmung bei einer Präzision von Laboratorium zu Laboratorium mit Variationskoeffizienten für die Phosphorwolframat-Methode von 9,8, 'einem solchen für die Uricase-Spaltung $(\mathrm{pH} 9,5)$ von 8,0 und einem Variationskoeffizienten für alle übrigen Methoden von 9,9 an der unteren Skala der brauchbaren und von Laboratorium zu Laboratorium vergleichbaren Analysen. $27 \%$ aller Untersucher hatten Ergebnisse, die mehr als das 3 fache der Standard-Abweichung vom Sollwert abweichen. Uber ähnlich schlechte Ergebnisse berichten KAISER et al. (1), die 1970 anläßlich einer interlaboritären Qualitätskontrolle in Österreich hinsichtlich der Harnsäure gefunden wurden: 12 von 25 Laboratorien lagen außerhalb des $\pm 10 \%$-Bereiches des Sollwertes.

Im wesentlichen stehen uns zwei verschiedene Methoden zur Bestimmung der Harnsäure zur Verfügung:

1. die Phosphorwolframsäure-Methode, die Folin und DeNIs (2) aus der Phosphormolybdänsäure-Methode von OfFER (3) entwickelten; diese Methode liegt den Modifikationen von HeILMEYeR und KREBS (4) sowie Caroll et al. (5) zugrunde.
2. enzymatische Methoden mit Uricase, die auf erste Berichte von KalCKAR und SchafraN (6) zurückführen. Die bisher gebräuchlichsten Techniken beruhen auf Arbeitsvorschriften von Praetorius und Poulsen (7) im Modifikationsvorschlag von MAHLER $(8,9)$ nach KortüM und KrING (10) (Beimischung von Glycerin zum Blindwert) oder DA FonseCA-Wollheim (11) (niedrigere Enzymkonzentration). Eine Weiterentwicklung des UV-Testes als Farbtest gelang KAGEYAMA (12), über welche erste Erfahrungen bereits veröffentlicht wurden $(13,14)$. Der Beckman-Analyzer mißt die $\mathrm{O}_{2}$ Umsetzungsgeschwindigkeit bei der unter Uricaseeinfluß ablaufenden Reaktion von Harnsäure zu Allantoin (15).

\section{Methoden}

Eine übersichtliche Zusammenfassung ist in Tabelle 1 gegeben.

\section{Reduktionsmethoden}

Die Reduktion von Phosphorwolframsäure im alkalischen Medium durch Harnsäure (FoLIN und DENIs (2)) ist Grundlage aller reduktometrischen Harnsäurcbestimmungen. Dic Extinktion des entsprechenden blauen Farbstoffs ist der Harnsäurckonzentration proportional und kann im Bereich 560 bis $720 \mathrm{~nm}$ gemessen werden. Hicrauf baut auf die Phosphorwolframat-Methode

a) nach Heilmeyer und Krebs (4) soivie

b) der Testsatz AccUric') (Carolt (5)).

Einc Enteiweißung des Serums ist in beiden Fällen notwendig. Sic geschicht bei der ersten Methode durch Uranylacetat, bei AccUric durch Phosphorwolframsäure, womit der nächste Schritt, die Oxidation der Harnsäure, glcich miteinbezogen wird. Für die Bcstimmung nach Herlmeyse und Krens (4) ist eine Filtration des Ansatzes vorgeschrieben, bei AccUric wird das ausgefällte Eiwciß abzentrifugiert. Abschließend wird der Ansatz durch Zugabe von 1) Testpackung der Fa. Gödecke, Frciburg. 
Tab. 1

Durchführungskriterien der 6 verschiedenen, getesteten Harnsäure-Methoden (vgl. auch „Arbeitsvorschriften") *RT = Raumtemperatur

\begin{tabular}{|c|c|c|c|c|c|c|c|c|c|c|c|c|c|c|c|c|}
\hline & Methode & 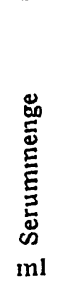 & 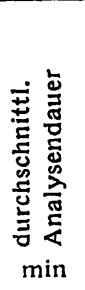 & 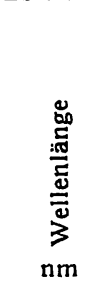 & min & 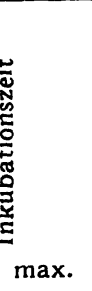 & 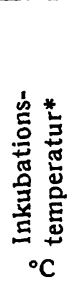 & 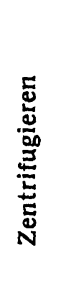 & 离 & 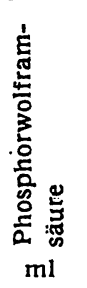 & 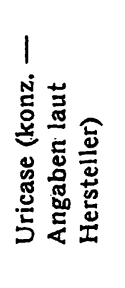 & · & 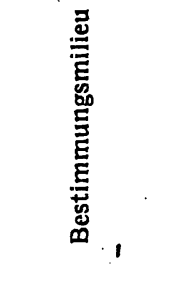 & 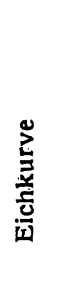 & 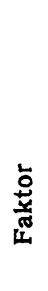 & 哥 \\
\hline A & $\begin{array}{l}\text { Phosphorwolframsäure } \\
\text { HEILMEYER }\end{array}$ & 2 & 20 & 578 & 10 & 30 & RT & $\varnothing$ & + & 0,2 & $\varnothing$ & $\varnothing$ & $\mathrm{Na}_{2} \mathrm{CO}_{3}$ & + & $\varnothing$ & $\varnothing$ \\
\hline B & $\begin{array}{l}\text { AccUric Macro } \\
\text { CAROLL }\end{array}$ & 0,2 & 40 & $\begin{array}{r}560 \\
-720\end{array}$ & 15 & 30 & RT & + & $\varnothing$ & 2 & $\varnothing$ & $\varnothing$ & $\mathrm{Na}_{2} \mathrm{CO}_{3}$ & + & + & $\varnothing$ \\
\hline c & $\begin{array}{l}\text { AccUric Micro } \\
\text { Gödecke } \\
\text { CAROLL }\end{array}$ & 0,05 & 40 & $\begin{array}{r}560 \\
-720\end{array}$ & 15 & 30 & $\mathrm{RT}$ & + & $\varnothing$ & 0,5 & $\varnothing$ & $\varnothing$ & $\mathrm{Na}_{2} \mathrm{CO}_{3}$ & + & + & $\varnothing$ \\
\hline D & $\begin{array}{l}\mathrm{O}_{2} \text {-Verbrauch } \\
\text { Beckman }\end{array}$ & 0,05 & 1 & $\varnothing$ & $\varnothing$ & $\varnothing$ & $\begin{array}{r}34 \\
\pm 1\end{array}$ & $\varnothing$ & $\varnothing$ & $\varnothing$ & $\begin{array}{c}1 \\
\mathrm{mg} / \mathrm{ml}\end{array}$ & $\varnothing$ & pH 2 & $\varnothing$ & $\varnothing$ & + \\
\hline $\mathrm{E}$ & $\begin{array}{l}\text { Uricase-UV-Test } \\
\text { KORTÜM }\end{array}$ & 0,2 & 30 & $\begin{array}{l}293 \\
297\end{array}$ & 10 & 10 & 25 & $\varnothing$ & $\varnothing$ & $\varnothing$ & $\begin{array}{c}2 \\
\mathrm{mg} / \mathrm{ml}\end{array}$ & + & $\begin{array}{c}\text { Borat } \\
\text { pH } 9,5\end{array}$ & $\varnothing$ & + & $\varnothing$ \\
\hline$F$ & $\begin{array}{l}\text { Urica-quant } \\
\text { KAGEYAMA }\end{array}$ & 0,5 & 45 & 405 & 30 & 120 & 37 & $\varnothing$ & $\varnothing$ & $\varnothing$ & $\begin{array}{c}5 \\
\mathrm{U} / \mathrm{ml}\end{array}$ & + & $\begin{array}{l}\text { Ammonium- } \\
\text { phosphat } \\
\text { pH } 7\end{array}$ & $\varnothing$ & $\varnothing$ & + \\
\hline
\end{tabular}

$\mathrm{Na}_{2} \mathrm{CO}_{3}$ alkalisiert. Die Reduktion der Phosphorwolframsäure ist unspezifisch und kann auch von anderen reduzierenden Substanzen, wie z. B. Ascorbinsäure, beeinflußt werden.

\section{Enzymatische Reaktion}

Auf einer spezifischen enzymatischen Reaktion beruht die Spaltung der Harnsäure durch Uricase (Urate: Oxygen oxidoreductase, EC 1.7.3.3) in Allantoin und Wasserstoffperoxid (PraEtorius und Poulsev (7)):

$$
\text { Harnsäure }+\mathrm{H}_{2} \mathrm{O}+\mathrm{O}_{2} \stackrel{\text { Uricase }}{\longrightarrow} \text { Allantoin }+\mathrm{CO}_{2}+\mathrm{H}_{2} \mathrm{O}_{2}
$$

Im für Harnsäure spezifischen Bereich von $293 \mathrm{~nm}$ kann die Abnahme der Harnsäurekonzentration direkt gemessen werden. Dieser UV-Test ${ }^{2}$ ) wurde zuletzt durch

a) KoRTüM und KIING (10) dadurch verbessert, daß der „Glycerinfehler" durch Zusatz einer der Uricaselösung entsprechenden Glycerinmenge zur Blindlösung vermieden wurde.

b) von Kageyama (12) wurde dieser Test 1972 zu einem colorimetrischen Test weiterentwickelt: das durch Harnsäurespaltung mittels Uricase entstehende Wasserstoffperoxid oxidiert mit Hilfe von Katalase Methanol zu Formaldehyd, welches wiederum bei Vorhandensein von Ammonium-Ionen mit Acetylaceton eine gelbgefärbte Verbindung bildet. Die Extinktion zwischen 400 und $420 \mathrm{~nm}$ ist der Harnsäurekonzentration proportional. Die Methode liegt dem Testansatz Urica-quant ${ }^{3}$ ) zugrunde.

c) die Messung der Harnsäurekonzentration mit dem BeckmanAnalyzer Modell ERA 2001) basiert auf der Messung des Sauerstoffverbrauchs während der enzymatischen Oxidation in Gegenwart von Uricase mit Hilfe einer polatographischen Sauerstoffelektrode. Als Meßgröße dient die Abnahmegeschwindigkeit der Sauerstoffkonzentration während der Katalyse, wobei der Gipfelwert bereits nach 10 Sekunden erreicht wird, lange vor dem Endwert der enzymatischen Reaktion. Der Sauerstoffverbrauch, welchem die Harnsäurekonzentration in der Probe proportional ist, wird elektronisch umgerechnet und liefert den Meßwert in $\mathrm{mg} /$ $100 \mathrm{ml}$ Harnsäure bezogen auf den Eichstandard. Probe und Uricasereagenz müssen auf $34^{\circ} \mathrm{C}$ erwärmt werden.

\section{Arbeitsvorschriften}

Diese entsprechen entweder der Originalliteratur (A), den Betriebsvorschriften der Gerätehersteller (D) oder den Reagenzien-Begleit-

2) Testpackung der Fa. Boehringer, Mannheim.

3) Testpackung der $\mathrm{Fa}_{2}$. Boehringer, Mannheim.

4) als Harnsäure-Bestimmungszusatz 652523 zum Glucose-Analyzer der Fa. Beckman, München. zetteln (B, C, E und F). Auf Modifikationen wurde bewußt verzichtet.

\section{A) Phosphorivolframat-Methode nach Heilmeyer und KReBS}

1. $2 \mathrm{ml}$ Serum $+2 \mathrm{ml} \mathrm{1,55}$ proz Uranylacetatlösung $+6 \mathrm{ml} \mathrm{H}_{2} \mathrm{O}$

2. Gut durchschütteln und Eiweiß abfiltrieren.

3. $4 \mathrm{ml}$ des klaren Filtrats $+0,2 \mathrm{ml}$ Phosphorwolframsäurereagenz (Folin-Denis) (100 g/1 Natriumwolframat, $80 \mathrm{ml} / 1$ o-Phosphorsäure) $+1,8 \mathrm{ml} 22$ proz Sodalösung.

4. Frühestens nach 8 , spätesten, nach 20 min mit Filter $578 \mathrm{~nm}$ gegen dest. Wasser bei Schichtdicke $2 \mathrm{~cm}$ photometrieren. Berechnung nach Eichkurve.

\section{$\mathrm{B}+\mathrm{C})$ AccUric Macro (Micro)}

Die Angabe für die Micro-Methode jeweils in Klammern:

1. Phosphorwolframsäure-Reagenz $2 \mathrm{ml}(0,5 \mathrm{ml})+$ Serum $0,2 \mathrm{ml}$ $(0,05 \mathrm{ml})$ gut mischen und $15 \mathrm{~min}$ bei Raumtemperatur stehen lassen.

2. Zentrifugieren bei $3000-5000 \mathrm{U} / \mathrm{min}$ für $3 \mathrm{~min}$ (ca. 15000 $\mathrm{U} / \mathrm{min}$ für $2 \mathrm{~min}$ ).

3. Macro: Uberstand sofort in ein Reagenzglas abgießen (Micro: $0,2 \mathrm{ml}$ des Uberstandes in ein Reagenzglas pipettieren).

4. Natriumcarbonatlösung $1 \mathrm{ml}(0,1 \mathrm{ml})$ zufügen, gut mischen und $15 \mathrm{~min}$ bei Raumtemperatur stehen lassen.

5. Extinktion bei einer Wellenlänge zwischen 560 und $720 \mathrm{~nm}$ gegen Wasser messen. Berechnung: Faktor oder Eichkurve.

\section{D) Beckman Analyzer}

1. Inkubation von Reagenz und Serumproben bei $34 \pm 1^{\circ} \mathrm{C}$.

2. Eichung des Gerätes durch Einpipettierung von $50 \mu \mathrm{l}$ Standardlösung in die Meßkammer.

3. $50 \mu \mathrm{l}$ der Probe werden in Meßkammer pipettiert. Das Ergebnis kann direkt in $\mathrm{mg} / 100 \mathrm{ml}$ abgelesen werden.

E) Uricase-Methode (Modifikation KorTüM und KLING)

1. Auf den Boden von Reagenzgläsern $0,2 \mathrm{ml}$ Serum $+10,0 \mathrm{ml}$ Boratpuffer $(100 \mathrm{~mol} / 1 ; \mathrm{pH} 9,5)$ pipettieren, mischen, verdünnte Probe $15 \mathrm{~min}$ bei $20-25^{\circ} \mathrm{C}$ stehen lassen.

2. Zum Leerwert $0,02 \mathrm{ml} 50$ proz Glycerin; zur Probe $0,02 \mathrm{ml}$ Uricase ( $2 \mathrm{mg}$ Uricase $/ \mathrm{ml} 50$ proz Glycerin) mischen, etwa $10 \mathrm{~min}$ bei 20 bis $25^{\circ} \mathrm{C}$ stehen lassen.

3. Messung in Quarzküvetten $1 \mathrm{~cm}$ Schichtdicke bei $293 \mathrm{~nm}$ gegen Leerwert. Berechnung: $c=68,4$ (Extinktion Probe + Extinktion Leerwert) $=\mathrm{mg} / 100 \mathrm{ml}$. 
F) Urica Quant

Auf den Boden von Reagenzgläsern pipettieren:

\begin{tabular}{|c|c|c|c|c|}
\hline & $\begin{array}{l}\text { Standard- } \\
\text { leerwert }\end{array}$ & Standard & $\begin{array}{l}\text { Proben- } \\
\text { leerwert }\end{array}$ & Probe \\
\hline Serum & - & - & $0,50 \mathrm{ml}$ & - \\
\hline $\begin{array}{l}\text { Standard }(6 \mathrm{mg} / 100 \mathrm{r} \\
\text { Harnsäure }\end{array}$ & $0,50 \mathrm{ml}$ & - & - & - \\
\hline $\begin{array}{l}\text { Harnsäure-Reagenz } \\
(0,48 \mathrm{~mol} / 1 \text { Ammo- } \\
\text { niumphosphat- } \\
\text { Puffer, pH } 7 \text {; } \\
2,5 \mathrm{~mol} / 1 \mathrm{Methanol} \text {; } \\
20 \mathrm{mmol} / 1 \text { Acetyl- } \\
\text { aceton; } 670 \mathrm{U} / \mathrm{ml} \\
\text { Katalase }\end{array}$ & $5,00 \mathrm{mi}$ & - & $5,00 \mathrm{ml}$ & - \\
\hline
\end{tabular}

Inhalt der Reagenzgläser gut mischen

Uricase $(5 \mathrm{U} / \mathrm{ml}$

50 proz Glycerin)

Aus dem Reagenz-

- $0,02 \mathrm{ml} \quad-\quad 0,02 \mathrm{ml}$

glas des Standard-

leerwertes

abpipettieren

Aus dem Reagenz-

glas des Proben-

leerwertes

abpipettieren

- 2,50 ml $\quad-\quad$

gut mischen, Leerwerte, Standard und Probe mindestens $60 \mathrm{~min}$ bei $37^{\circ} \mathrm{C}$ inkubieren.

Extinktion der Probe gegen den Probenleerwert (E $E_{\text {Probe }}$ ) und Extinktion des Standards gegen den Standardleerwert (Estandard) bei $405 \mathrm{~nm}$ in $1 \mathrm{~cm}$ Glasküvette messen.

Berechnung: $c=6 \frac{E_{\text {Probe }}}{E_{\text {Standard }}}[\mathrm{mg} / 100 \mathrm{ml}]$.

Zur Umrechnung von $\mathrm{mg} / 100 \mathrm{ml}$ in $\mu \mathrm{mol} / 1$ wird mit dem Faktor 59,48 multipliziert.

\section{Versuchsanordnung}

Als Testsubstanzen dienten uns

a) selbst eingewogene Eichlösungen. Aus einer $1190 \mu \mathrm{mol} / 1-\mathrm{Stamm}-$ lösung [20 mg Harnsäure (2,6,8-Trihydroxy-purin, $\mathrm{C}_{5} \mathrm{H}_{4} \mathrm{~N}_{4} \mathrm{O}_{3}$ MG 168, 11, der Fa. Fluka) wurden in $100 \mathrm{ml}$ Albuminlösung $(50 \mathrm{~g} / \mathrm{l})$ gelöst] wurden Eichlösungen von 119, 238, 357, 476, 595, 714, 833, 952, 1071 und $1190 \mu \mathrm{mol} / 1$ Harnsäure hergestellt.

b) käufliche Kontrollseren:

1. Precilip (Boehringer, Mannheim) C-Nr. 102, Sollwert 286 442,9 $\mu \mathrm{mol} / 1$.
2. Monitrol I (Merz \& Dade, USA) C-Nr. LTD-112 A, B, Sollwert $286 \pm 13,1 \mu \mathrm{mol} / 1$

3. Monitrol II (Merz \& Dade, USA) C-Nr. PTD-27A, Sollwert $506 \pm 20,2 \mu \mathrm{mol} / \mathrm{l}$.

4. Enzatrol (Merz \& Dade, USA) C-ET-237 A, B Sollwert $518 \pm 15,5 \mu \mathrm{mol} / 1$.

5. Seronorm (Nyegaard, Oslo) C-Nr. 118, Sollwert $339 \pm 47,0$ $\mu \mathrm{mol} / 1$.

6. Versatol (Warner-Lambert, USA) C-Nr. 2501079, Sollwert $190 \pm 15,5 \mu \mathrm{mol} / \mathrm{l}$

Zur Ermittlung der Präzision in der Serie wurde mit jeder Methode eine Zchnfach-Bestimmung einer jeden Eichkonzentration zwischen 119 und $1190 \mu \mathrm{mol} / 1$ durchgeführt.

Doppelbestimmungen einer $357 \mu \mathrm{mol} / 1$-Eichlösung sowie aller angegebenen Kontrollseren dienten an 10 verschiedenen Tagen zur Feststellung der Präzision von Tag zu Tag.

Die Abweichungen der Meßwerte vom Sollwert wurden in Prozent angegeben und ergaben einen Anhalt für die Richtigkeit der einzelnen Methoden.

Zur statistischen Auswertung der Meßreihen wurden Mittelwerte $(\bar{x})$, Standardabweichung (2s) und Variationskoeffizient (VK) errechnet. Eine Regressions-( $y=a x+b)$ und Korrelations (r)Analysc wurde durchgeführt, um die Übereinstimmung der mit den verschjedenen Methoden gefundenen Werte zu beurteilen.

\section{Ergebnisse}

Die Abbildungen 1-7 zeigen, in wieweit die mit den einzelnen Methoden gefundenen Werte im Bereich 119 bis $1190 \mu \mathrm{mol} / 1$ den Sollwerten entsprechen und bis zu welcher Konzentration eine lineare Abhängigkeit besteht.

Die Reduktionsverfahren ergeben im unteren Bereich allgemein höhere Werte (Abb. 1-3) als die enzymatischen (Abb. 4-6). Linearität über den ganzen Bereich zeigen nur die.Methode nach KortüM und die KAGEYAMA-Methode. AccUric-Macro verläuft bis etwa 900 $\mu \mathrm{mol} / \mathrm{l}$ linear (Abb. 1), die Micro-Methode ergibt unter $350 \mu \mathrm{mol} / \mathrm{l} \mathrm{zu}$ hohe, oberhalb davon zu niedrige Werte. Die Phosphorwolframat-Methode nach HeILMEYER zeigt bis zu $830 \mu \mathrm{mol} / \mathrm{l}$ Linearität; die Beckman-Methode bis etwa $700 \mu \mathrm{mol} / 1$. Für den praktisch wichtigen Bereich zwischen 250 und $700 \mu \mathrm{mol} / \mathrm{l}$ wurden bei den reduzierenden Methoden im Schnitt 4-5\% Abweichung vom Sollwert gefunden (Abb. 7). Die besten Ergebnisse zeigte sowohl hier als auch in der Präzision (Abb. 8) die
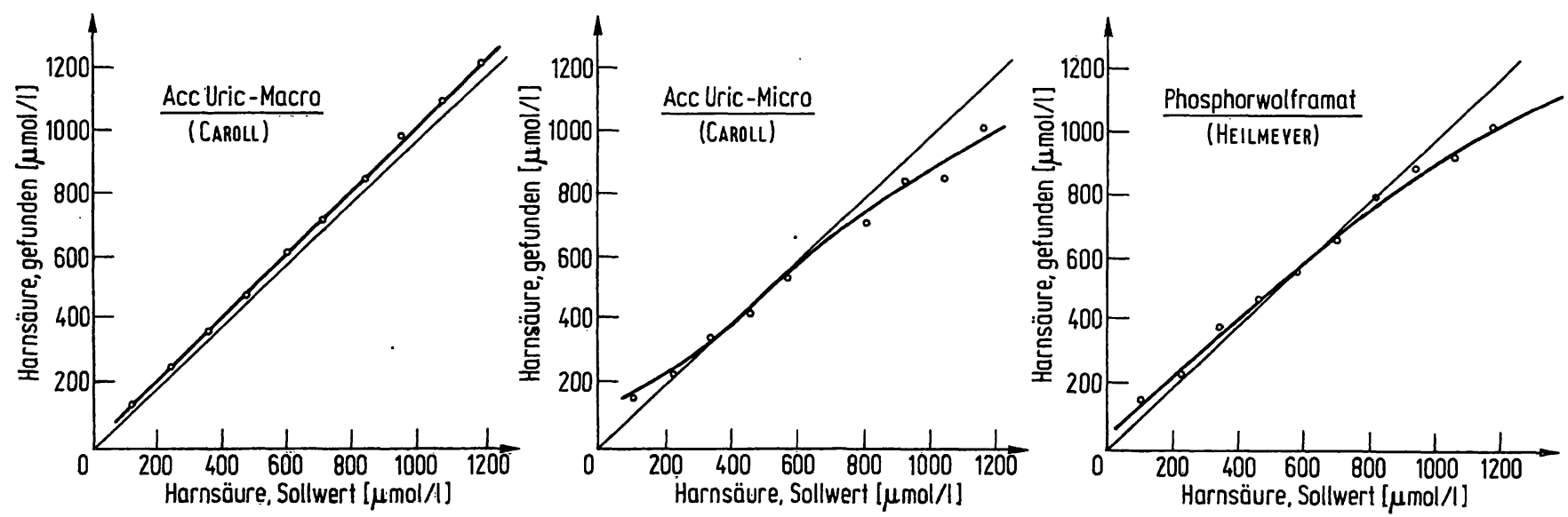

Abb. $1-3$

Vergleich der Sollwerte von 10 verschiedenen, eingewogenen Harnsäure-Konzentrationen zwischen 119 und $1190 \mu$ mol/l in Albuminlösung (50 g/l)

mit den Mittelwerten der gefundenen Werte aus 10-fach-Bestiminungen in der Serie
Phosphorwolframat-Methoden (AccUric-Macro und AccUric-Micro mit gleichzeitiger Pliosphorwolframat-Enteiweißung, Methode nach HEILMEYER mit Uranylacetat-Enteiweißung) 

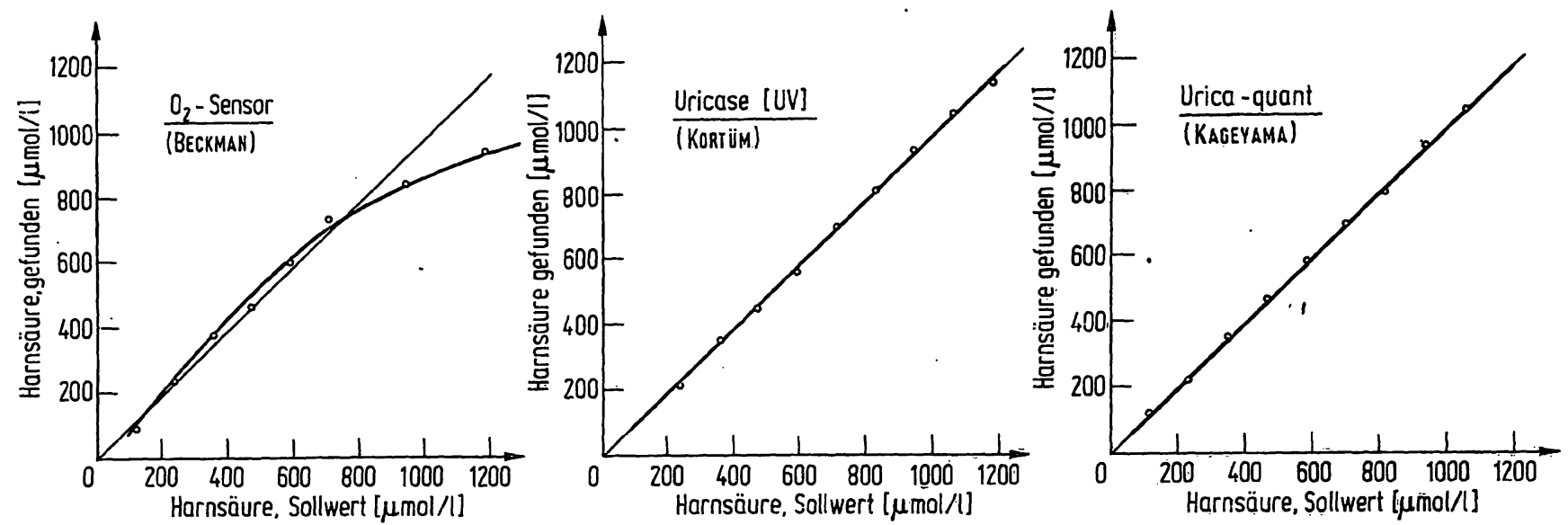

Abb. 4-6

Vergleich der Sollwerte von 10 verschiedenen, eingewogenen Harnsäure-Konzentrationen zwischen 119 und $1190 \mu$ mol/1 in Albuminlösung (50 g/l) mit den Mittelwerten der gefundenen Werte aus 10 -fach-Bestimmungen in der Serie

Uricase-Methoden (Sauerstoffmessung bei der Uricasespaltung mit einem $\mathrm{O}_{2}$-Sensor, Extinktionsabnahme bei $293 \mathrm{~nm}$, Uricase-Katalase-Farbreaktion)

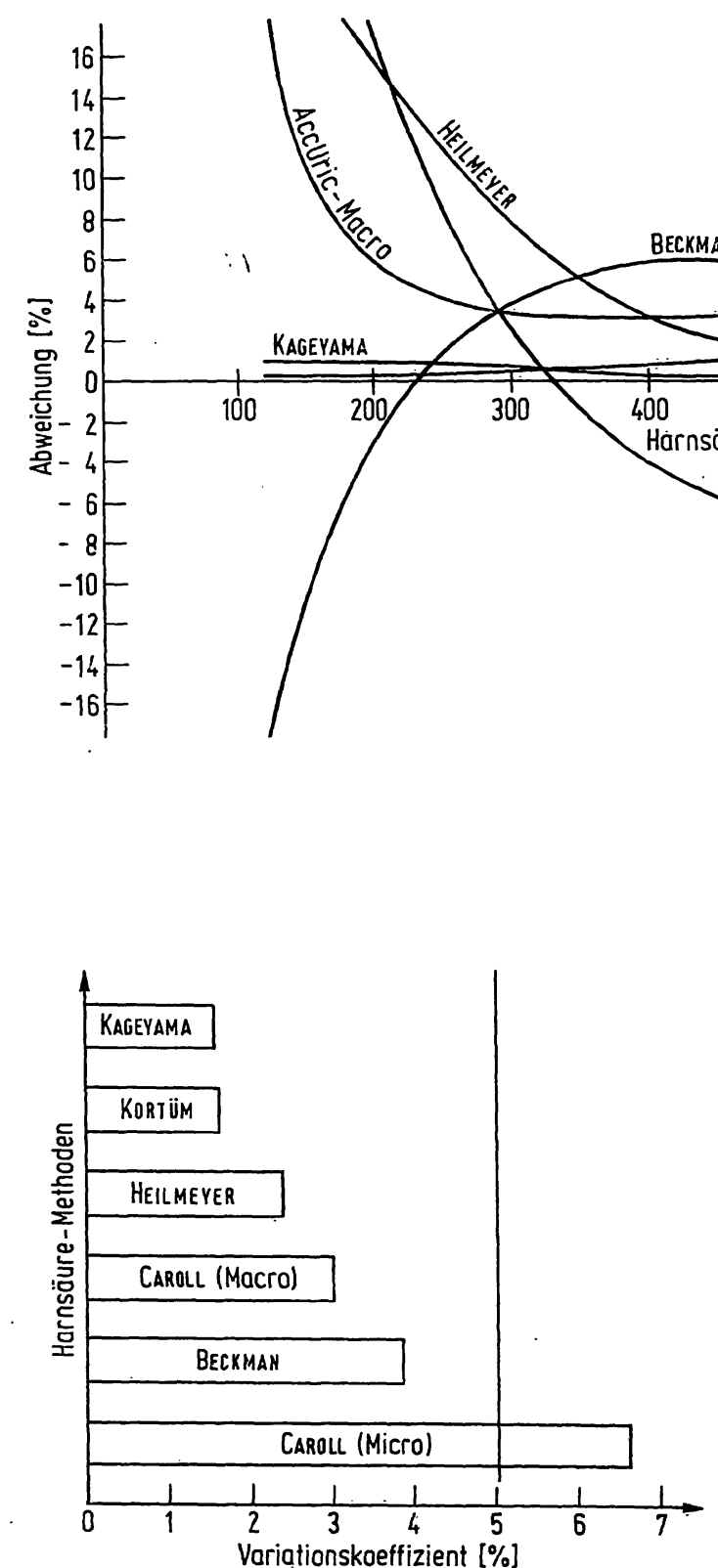

Abb. 8

Variationskoefffizienten 6 verschiedener Harnsäure-Methoden, bestimmt bei 10 -fach-Bestimmungen in der Serie in 10 verschiedenen Konzentrationsbereichen 2 wischen 119 und $1190 \mu \mathrm{mol} / 1$ eingewogener Harnsäure in Albuminlösung $(50 \mathrm{~g} / \mathrm{l})$
Abb. 7

Mittlere prozentuale Abweichung vom Sollwert bei serieller 10-fach-Bestimmung von eingewogenen Harnsäuresäurebestimmungen

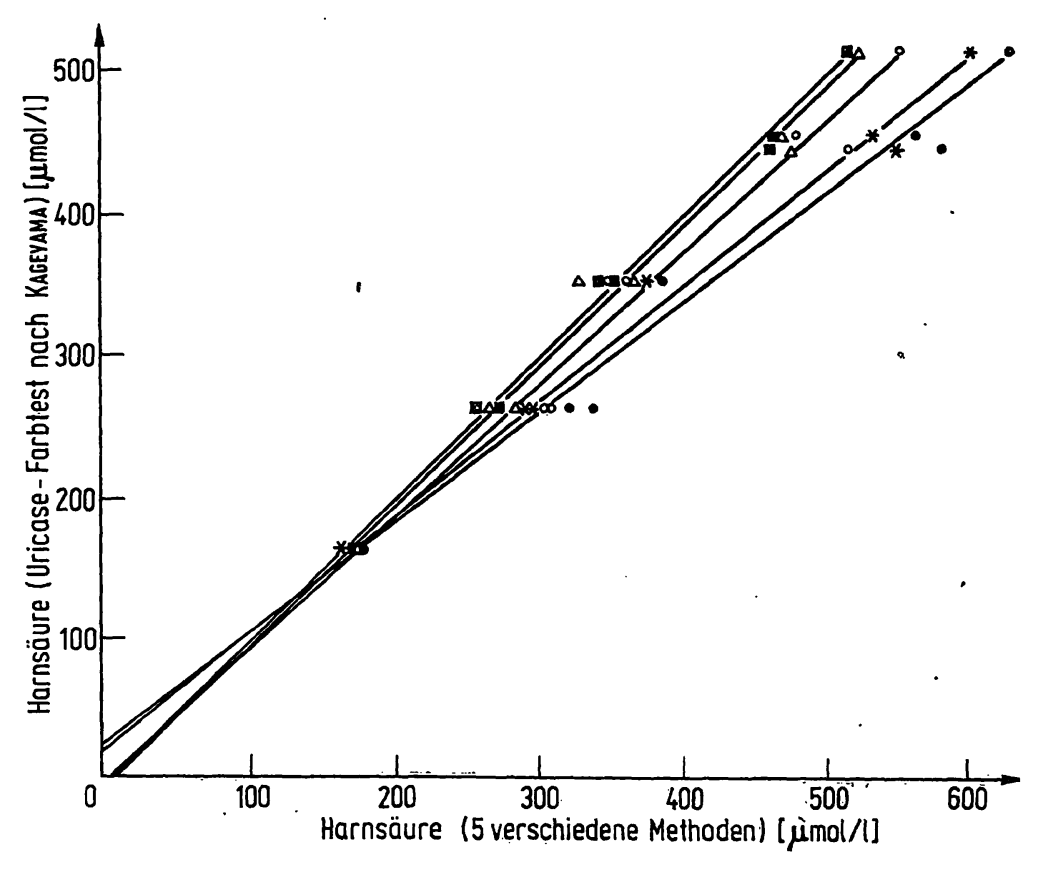

Abb. 9

Regressionsgeraden 5 verschiedener Harnsäure-Methoden im Vergleich zur kolori-. metrischen Harnsäure-Bestimmung nach KAGEYAMA. Zugrundeliegende Werte wurden als Mittelwerte aus Doppel-Bestimmungen von Tag zu Tag an 6 verschiedeKonzentrationen an 10 verschiedenen Tagen er-

$=$ Uricase-UV-Methode (KORTÜM), y =0,9978 x + 0,1452;

$=$ Uricase- $\mathrm{O}_{2}$-Sensor-Methode (Beckman), $\mathrm{y}=1,0068 \mathrm{x}+0,1803$

$=$ Phosphorwolframat-Methode (AccUric-Micro), $y=1,0704 \times+0,1138$;

Phosphorwolframat-Methode (HEILMEYER), $y=1,2802 \times-0,8371$.

$=$ Phosphorwolframat-Methode (AccUric-Macro), $y \stackrel{y}{=} 1,2776 x-0,4436$ 
Tab. 2

Vergleich der 6 verschledenen Harnsäure-Methoden untereinander an Hand von 10-fach Bestimmungen in der Serle bel 10 verschiedenen Konzentrationen zwischen 119 und $1190 \mu \mathrm{mol} / 1$ Harnsäure in Albuminlösung $(50 \mathrm{~g} / \mathrm{l})$

Korrelationskoeffizient $(r)=$ errechnet aus dem Vergleich der Mittelwerte verschiedener Harnsäure-Konzentrationen

Regressionskoeffizient (a) $\approx$ Steigung der Regressionsgeraden Regressionskonstante $(b)=$ Schnittpunkt der Regressionsgeraden mit der $\mathbf{x}$-Achse

\begin{tabular}{llccc}
\hline \multicolumn{1}{c}{$\mathbf{x}$} & \multicolumn{1}{c}{$\mathbf{y}$} & $\mathbf{r}$ & $\mathbf{a}$ & $\mathbf{b}$ \\
\hline AccUric Macro & AccUric Micro & 0,9952 & 0,7907 & 0,9745 \\
& HeILMEYER & 0,9975 & 0,8306 & 1,0320 \\
& Beckman & 0,9874 & 0,8265 & 0,8104 \\
& KORTÜM & 0,9961 & 0,9713 & 0,1798 \\
& KAGEYAMA & 0,9965 & 0,7705 & 0,1924 \\
AccUric Micro & HEILMEYER & 0,9937 & 1,0414 & 0,0999 \\
& Beckman & 0,9881 & 1,0409 & 0,1641 \\
& KORTÜM & 0,9941 & 1,2259 & 1,3166 \\
HEILMEYER & KAGEYAMA & 0,9951 & 1,2160 & 1,2746 \\
& Beckman & 0,9924 & 0,5559 & $0,2 a 75$ \\
& KORTÜM & 0,9964 & 1,1632 & 1,3209 \\
Beckman & KAGEYAMA & 0,9963 & 1,1638 & 1,2874 \\
& KORTÜM & 0,9883 & 1,1478 & 0,8486 \\
KORTÜM & KAGEYAMA & 0,9874 & 1,1453 & 0,8457 \\
& KAGEYAMA & 0,9988 & 0,9985 & 0,0065 \\
\hline
\end{tabular}

Kageyama-Methode. Die mit Abstand größte Streuung der Werte wurde bei AccUric-Micro gefunden. Abbildung 9 und Tabelle 2 zeigen die sehr gute Ubereinstimmung der Uricase-UV-Methode und der UricaseKatalase-Methode.

Die Doppelbestimmungen einer $357 \mu \mathrm{mol} / \mathrm{l}$-Eichlösung an 10 verschiedenen Tagen zeigen bei den reduzierenden Methoden und beim Beckman-Analyzer höhere Werte als bei der Uricase-Methode oder bei der UricaseKatalase-Methode. Die mit letzteren Methoden gefundenen Werte bieten hinsichtlich der Streuung sowie der Abweichung vom Sollwert die besten Ergebnisse, während die mit dem Beckman-Analyzer und AccUricMicro gefundenen Werte stark streuen $(2 s> \pm 60$ $\mu \mathrm{mol} / 1)$.

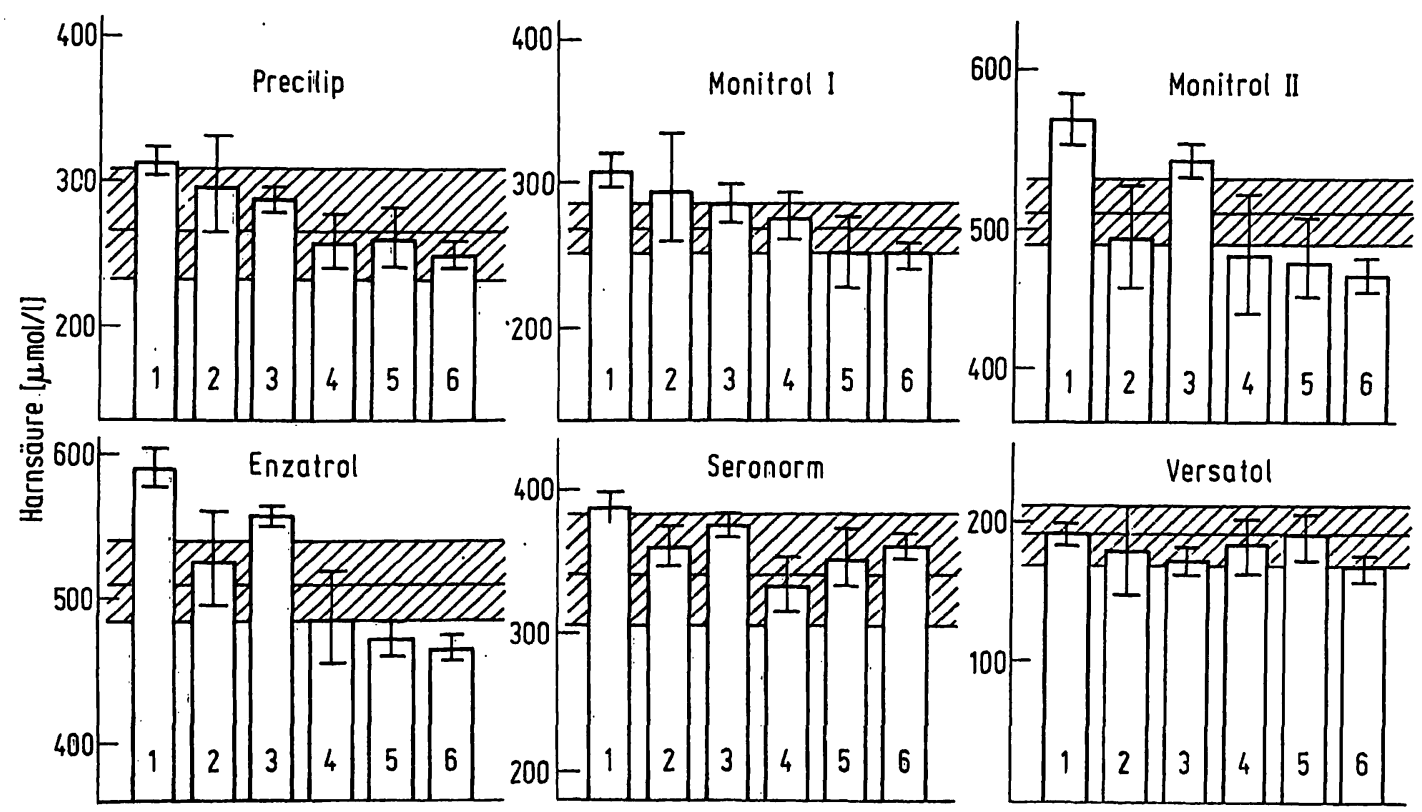

Abb. 11

Bestimmung 6 verschiedener, Harnsăure-haltiger, käuflicher Kontrollseren an 10 verschiedenen Tagen mit 6 verschiedenen Methodell.

Schraffierter Bereich: Der von den Firmen angegebenc Vertrauenbereich um den Sollwert
$(1$ - AccUric Macro; 2 - AccUric Micro; 3 - HEILMEYER; 4 - Beckman ; 5 - KORTUM; 6 - KAOEYAMA)

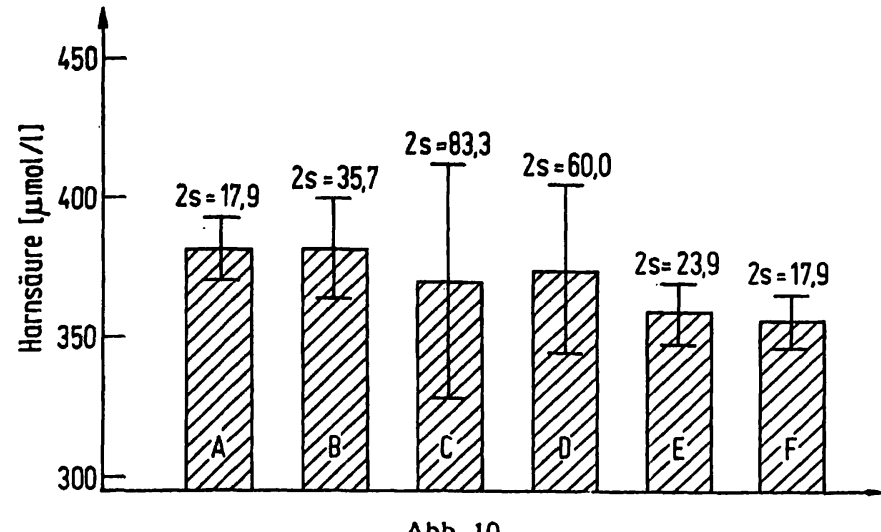

$357 \mu \mathrm{mol} / \mathrm{l}-\mathrm{Standard}$ an 10 verschiedenen Tagen mit 6 verschiedenen Methoden auf Harnsäure untersucht. Mittelwerte \pm 2 s errechnet aus

$$
\begin{aligned}
& A=\text { Phosphorwolframat-Methode (HEILMEYER) } \\
& B=\text { Phosphorwolframat-Methode (AcCUric-Macro) } \\
& C=\text { Phosphorwolframat-Methode (AccUric-Micro) } \\
& D=\text { Uricase-O,-Sensor (Beckman) } \\
& \text { E = Uricase-UV-Methode (KORTÜM) } \\
& F=\text { Uricase-Katalase-Methode (KAGEYAMA) (Vgl. Tab. 1) }
\end{aligned}
$$

Eine gute Reproduzierbarkeit weist die Methode nach Heilmeyer und Krebs auf $(2 \mathrm{~s}= \pm 17,9 \mu \mathrm{mol} / \mathrm{l}$ ) (Abbildung 10).

Ähnliche Ergebnisse hinsichtlich der Reproduzierbarkeit und der Streuung können in der Tag-zu-Tag-Serie (10 verschiedene Tage; Doppelbestimmungen) bei den unterschiedlichsten käuflichen Kontrollseren gefunden werden (Abb. 11). Nur werden mit den durchgeführten Methoden sehr unterschiedliche Ergebnisse hinsichtlich der Richtigkeit erzielt. Während beim Versatol und Seronorm mit Hilfe aller durchgeführten Methoden die Abweichung vom Sollwert sehr gering ist, werden z. B. bei Monitrol II und Enzatrol sehr unterschiedliche Abweichungen festgestellt. Um $10 \%$ zu hohe Werte durch die AccUric-Macro-Methode stehen um $12 \%$ zu niedrige Werte durch die Urica-quant-Methode bei Kontrollen mit Enzatrol gegenüber. Dic Reduktionsverfahren brachten im allgemeinen höhere Werte. 


\section{Diskussion}

Im Grunde genommen erfüllen alle getesteten Methoden mit Ausnahme der AccUric-Micro-Methode die Bedingungen, die an eine strapazierfähige Routinemethode für die Bestimmung der Harnsäure gestellt werden müssen (VK zwischen 1,6\% für die Uricase-KatalaseMethode und 3,7\% für Beckman-Analyzer). Die besten Ergebnisse liefert auch in Hinsicht der Handhabung die Methode nach KageYama. Es besteht eine ausgezeichnete Korrelation zu der bisher als Referenz-Methode üblichen Uricase-UV-Methode $(r=0,998)$. Diese Ergebnisse stimmen mit denen von MerTz ( $r=0,9976)$ voll überein. Die Methode nach KageYama zeichnet sich im Vergleich zur UV-Methode jedoch durch noch bessere Reproduzierbarkeit aus (Tab. 2).

Die Ergebnisse mit dem Beckman-Analyzer sind wesentlich schlechter. Hier wird der sehr geringe Zeitaufwand für die Analyse sowie die geringe Probenmenge durch erhebliche Instabilität der Analysenergebnisse kompensiert. Trotz Einhaltens möglichst exakter Temperaturkonstanz weichen nach bereits 5 bis 10 Messungen die Meßwerte nach oben hin merklich ab, und es wird eine Korrektur des Standard-Sollwertes am Gerät notwendig. Dadurch erhöhen sich Zeitaufwand und die Kosten pro Analyse erheblich. Außerordentlich wichtig ist ein exaktes Einhalten der Temperatur sowohl beim Reagenz als auch bei der Serumprobe. Durch den schwer einstellbaren Beckman-Inkubator für das Serum kommt es oft zu Temperaturschwankungen mit beträchtlich veränderten Werten.

AccUric-Macro und die Phorphorwolframat-Methode nach HeILMEYer zeigen im Gegensatz zu enzymatischen Methoden durchweg höhere Werte (ähnlich den Ergebnissen von RetTENBACHeR (16) zwischen 53,6 bis 69,0 $\mu \mathrm{mol} / \mathrm{l})$, jedoch zeichnen sie sich durch austeichende Reproduzierbarkeit aus. Die Micro-Methode hingegen erweist sich als am wenigsten zuverlässig. Die kleinen Mengen führen zu einer starken Erhöhung der zufälligen Fehler. Die Gefahr von Verschleppungsfehlern ist bei den schwer zu reinigenden Micro-Küvetten besonders groß. Dabei ist bei allen drei Reduktionsverfahren eine so sorgfältige Reinigung der Küvetten nach jeder Messung notwendig, da Natriumcarbonatrückstände als Kristallisationskeime dienen. Es werden zum Teil erhebliche Extinktionsdifferenzen vor und nach Reinigung der Küvetten gefunden. Die Analysendauer wird unter diesen Bedingungen nicht unerheblich verlängert.

Bei der Methode nach HeIlmeyer und KREBs ist es oft schwierig, ein Eiweiß-freies, klares Filtrat zu bekommen. Der Arbeitsaufwand, bedingt durch 6 Pipettiervorgänge und das Filtrieren, ist höher als bei allen anderen Methoden.

Der UV-Test hat laut Testpackungsvorschriften gegenüber der Uricase-Katalase-Methode den Nachteil, daß ein exaktes Arbeiten nach Stoppuhr und große Arbeitskonzentration erforderlich und eine Beschränkung auf 10 Proben in einer Arbeitsserie unumgänglich wäre. Jedoch konnten wir bei Überprüfung der Zeitabhängigkeit der Uricasespaltung im UV-Test bei frischen Reagenzien eine stabile Reaktion bereits nach der 6. Minute feststellen. Im beobachteten Zeitraum über eine Stunde konnten lediglich Extinktionsschwankungen $<0,001$ festgestellt werden. Ein gefordertes, exakt zeitgebundenes Ablesen der Extinktion ist also nicht erforderlich. Der Arbeitsaufwand ist somit nicht entscheidend größer als bei der KAGEYAMA-Methode. Es muß allerdings erwähnt werden, daß die erwähnte Stabilität der Reaktion nur bei absolut frischen Lösungen festgestellt werden konnte. Stabiler waren die Reaktionen bei der Uricase-Katalase-Methode: Hier konnte noch bis zu 11/2 Monaten eine gleiche Farbentwicklung in der Zeiteinheit festgestellt werden, die von der 30. Minute ab stabil war und innerhalb des Beobachtungszeitraums von 2 Stunden stabil blieb. 3 Monate nach Ansetzen des die Katalase enthaltenden Reagenzes waren zufriedenstellende Ergebnisse nicht mehr zu erzielen.

Lediglich diese Überlegungen sowie die Bedienung eines Spektralphotometers für die UV-Methode und weniger die nur gering schlechtere Reproduzierbarkeit machen den UV-Test der Uricase-Katalase-Methode geringgradig unterlegen.

\section{Danksagung}

Wir danken den Firmen Beckman (München), Boehringer (Mannheim) und Gödecke (Freịburg), die uns Testpackungen und Reagenzien für diese Untersuchungen zur Verfügung gestellt haben.

\section{Literatur}

1. Kaiser, E., Schreiber, V., Bauer, P., Havelec, L., Wohlzogen, F. X. \& GABL, F. (1972), Wien. Klin. Wochenschr. 84, 217-219. 2. Folin, O. \& DenIs, W. (1912/1913), J. Biol. Chem, 13, 469-472. 3. Offer, T. R. (1894), Centr. Bl. Physiol. 8, 801. - 4. HeILMEYER, L. \& KREBS, W. (1930); Biochem. Z. 223, 352-365. - 5. Caroll, J. J., Coburn, H., Douglas, R. \& Babson, A. L. (1971), Clin. Chem. 17, 158-160. - 6. KalCKar, H. M. \& Shafran, J. (1947), J. Biol. Chem. 167; 429-433. - 7. Praetorius. E. \& Poulsen, H. (1953), Scand. J. Clin. Lab. Invest. 5, 273-280. 8. MAHLER, J. L. (1970), Anal. Biochem. 38, 65-84. - 9. MAHLER,
J. L. (1972), Ärztl. Lab. 18, 451. - 10. KortüM, M. \& KLING, O. (1971), Ärztl. Lab. 18, 33-36. - 11. DA FonseCA-WolLherm, F. (1972), diese Z. 10, 136 - 12. Kageyama,..N. (1971), Clin. Chim. Acta 31, 421-426. - 13. Mertz, D. P. (1973), Klin. Wochenschr. 51, 96-98. - 14. Thefeld, W., HofpMeIster, H., Busch, E. W., Koller, P. H. \& Vollmar, J. (1973), Deut. Med. Wochenschr. 98, 380-384. - 15. Beckman Instructions 83591; Beckman Instruments Fullerton USA, 1971. - 16. RETTENBACFER, F. (1972), Wien. Klin. Wochenschr. 84, 214-216.

PD Dr. J. D. Kquse-Jarres Chirurg. Univ.Klinik Klin. Chemie 78 Freiburg/Br. Hugstetterstraße 55 